\section{Bienen helfen Allergikern}

n niederländischen Paprika-Gewächshäusern haben über die Hälfe der Arbeiter respiratorische Symptome, 35\% sind gegen Paprikapollen sensibilisiert. Da die Arbeiter nur ungern Schutzmasken tragen, wurde in einer Studie als Alternative der pollenreduzierende Effekt von Bienen untersucht, die man dazu in den Gewächshäusern aussetzte. Die Insekten sammelten tatsächlich fleißig Paprikapollen, es ließ sich sogar eine inverse Korrelation zwischen der Anzahl der Bienen und den Pollenbelastung feststellen. Parallel dazu gingen auch die nasalen Symptome bei den Gewächshausarbei-

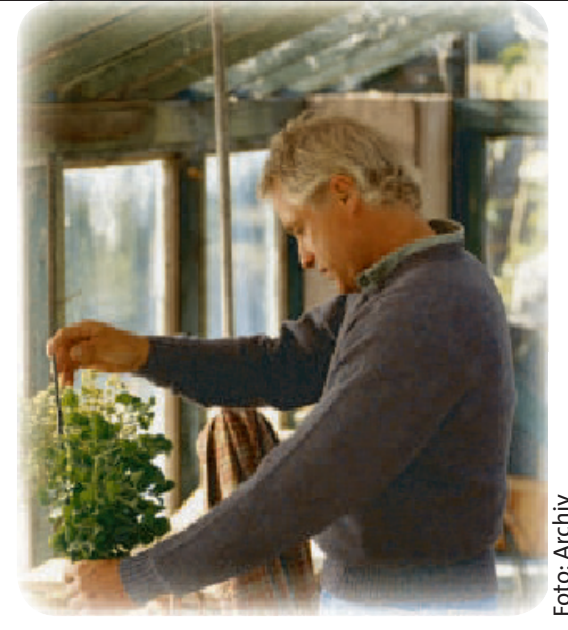

tern zurück. Die Bienen verhielten sich in der künstlichen Umgebung weitgehend friedlich.

de Jong NW et al. Int Arch Allergy Immunol 2006; 141: 390-5

\title{
Allergennitrierung als Allergietrigger
}

P

osttranslationale Veränderungen der Proteinstruktur haben häufig Veränderungen der Immunreaktivität gegen die Eiweiße zur Folge. Solche Reaktionen werden teilweise in vivo vom Organismus selbst induziert, z. B. indem im Rahmen von Entzündungsreaktionen durch Proteinnitrierung die Immunantwort gesteigert wird. Aber auch eine ex vivo stattfindende Nitrierung kann die Immunreaktivität von Proteinen erhöhen. Klinisch relevant wird dies bei der Nitrierung von Pollenallergenen durch hohe Stickstoffdioxidkonzentrationen in Anwesenheit von Ozon als Co-Faktor. Derart modifizierte Proteine triggern im Experiment verschiedene Parameter der Immunantwort. So erhöht nitriertes Bet v 1a in sensibilisierten Mäusen gegenüber dem nativen Birkenpollenhauptallergen die Produktion von spezifischem IgE sowie von IL-5 und Interferon- $\gamma$. Die Nitrierung von Allergenen könnte ein Grund für den Anstieg allergischer Erkrankungen sein.

$b k$

Gruijthuijsen YK et al. Int Arch Allergy

Immunol 2006; 141: 265-75

\section{Kreuzreagierende Nüsse}

Datienten mit einer Erdnussallergie sind oft auch gegen andere Nüsse sensibilisiert. Die verantwortlichen Allergene sind allerdings noch nicht identifiziert. In einer Experimentalstudie wurde deshalb das wichtigste Erdnussallergen, Ara h 2, näher untersucht. Ara h 2 ist ein Glykoproteinallergen, das Homologien zu Conglutin-Proteinen aufweist, die vielen Pflanzen als Speicherproteine dienen. Die Seren Erdnuss-allergischer Patienten mit hohem spezifischem IgE gegen Ara h 2 wurden mit steigenden Konzentrationen von Mandel- bzw. Paranussextrakt präinkubiert und anschließend ein Inhibitions-ELISA mit rekombinantem rAra h 2 durchgeführt. Ergebnis: Die zugegebenen Extrakte inhibierten die IgE-Bindung von $r$ Ara h 2. Dies weist auf gemeinsame IgE-Bindungsepitope von Ara h 2 und Allergenen von Mandel oder Paranuss hin.

$b k$

de Leon MP et al. Mol Immunol 2007; 44: 463-71

\section{Abwechslung gesucht}

ür untreue Frauen gibt es jetzt mög-
licherweise eine gute Ausrede: „Lieb-
ling, unser Immunsystem ist zu ähnlich!“
Forscher aus New Mexico fanden heraus,
dass Frauen, deren Immunsystem große
Ähnlichkeit zu dem ihres Partners auf-
weist, am ehesten zu Seitensprüngen nei-
gen. Bei Männern war ein solcher Zusam-
menhang dagegen nicht feststellbar. de

Garver-Apgar CE et al. Psychol Sci 2006;

17: 830-5

\section{Saurer Atem}

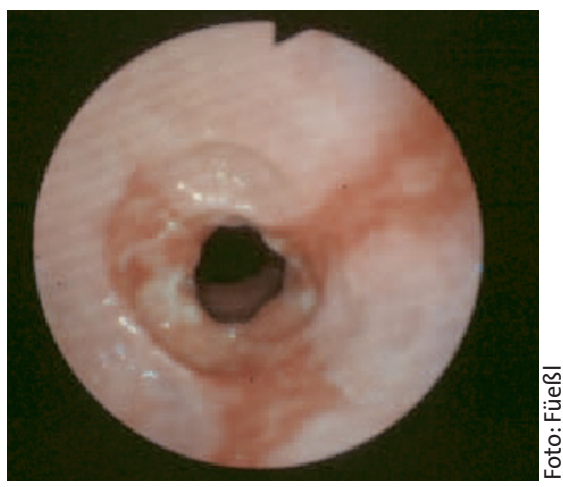

astroösophagealer Reflux verschlechtert die Asthmasymptomatik. Ob säureblockierende Maßnahmen auch der Lunge helfen, ist noch nicht ganz klar. Möglicherweise muss der Magen-pHWert bei diesen doppelt erkrankten Patienten besonders drastisch angehoben werden, so das Ergebnis einer kleinen Studie an 30 Patienten mit Asthma und gastroösophagealem Reflux, die randomisiert entweder einen $\mathrm{H}_{2}$-Blocker oder einen Protonenpumpenhemmer erhalten hatten. Beide Medikationen reduzierten den Reflux um durchschnittlich 90\%, aber nur der Protonenpumpenhemmer verbesserte auch Lungenfunktion und Asthmakontrolle. Offensichtlich sind nur die stärker säureblockierend wirkenden Protonenpumpenhemmer in der Lage, die Refluxbedingte Ansäuerung der Atemluft und die damit verbundene bronchiale Entzündung zurückzudrängen.

Shimizu Y et al. Tohoku J Exp Med 2006; 209: 181-9 